\title{
Polymer matrix materials selection for short sugar palm composites using integrated multi criteria evaluation method
}

\begin{abstract}
This work introduces a novel model for prioritizing and evaluating polymer matrix materials using integrated multi-criteria evaluation method. The selection process determines the most suitable polymer for incorporating into short sugar palm fiber composites. The prioritization procedure is accomplished using three different selection methods: AHP, TOPSIS, and ELECTRE. AHP is used for prioritizing alternatives. TOPSIS and ELECTRE attain the ranking analysis of alternatives on different scales. A novel tripartite analysis is developed as a new integrative approach for ranking the three selection methods. The analysis supports relevant comparisons and determines the correlations between prioritized alternatives and main criteria.
\end{abstract}

Keyword: AHP; TOPSIS; ELECTRE; Multi-criteria evaluation; Tripartite analysis; Composite materials 